\title{
Cerebral Venous Thrombosis in the Superior Sagittal Sinus as a Rare Cause of a Paroxysmal Kinetic Tremor
}

\author{
Kei Murao ${ }^{a}$ Shuji Arakawa ${ }^{a}$ Yoshihiko Furuta $^{a}$ Masahiro Shijo $^{b}$ \\ Tetsuro Ago $^{\text {b }}$ Takanari Kitazono $^{\text {b }}$ \\ ${ }^{a}$ Department of Cerebrovascular Diseases, Japan Organization of Occupational Health \\ and Safety, Kyushu Rosai Hospital, Fukuoka, Japan; ${ }^{b}$ Department of Medicine and Clinical \\ Science, Graduate School of Medical Sciences, Kyushu University, Fukuoka, Japan
}

\section{Keywords}

Cerebral venous thrombosis · Kinetic tremor · Involuntary movement

\begin{abstract}
Cerebral venous thrombosis (CVT) has a broad spectrum of clinical presentation compared to arterial etiology. Seizure is one of the common symptoms and is more frequent than in other stroke types. Hence, transient neurological symptoms in CVT patients are usually due to epileptic seizures, while transient repetitive movement disorder is extremely rare except as a complication of epilepsy. We report a case of CVT in the superior sagittal sinus with a 1-year history of paroxysmal kinetic tremor without evident epilepsy.
\end{abstract}

\section{Case Presentation}

A 60-year-old, right-handed male with involuntary shaking movements of the rightsided extremities visited our service. These episodes resolved within $5 \mathrm{~min}$ and were restricted to the extremities of the right side. He complained that the attacks were elicited by 


\section{Case Reports in Neurology}

Murao et al:: Cerebral Venous Thrombosis in the Superior Sagittal Sinus as a Rare Cause of a Paroxysmal Kinetic Tremor

using the right hand or foot when for instance writing, tightening a bolt with a driver, and fitting shoes, but not by postural changes. A personal note he wrote with his right hand during an attack proved an intermittent slight trembling movement (Fig. 1). He was always alert, and no other neurological symptoms arose during the course. Neurological examination at the first visit did not show any abnormal findings such as cerebellar ataxia and extrapyramidal signs. He had a history of hypertension but no other diseases leading to hypercoagulability such as infection, head trauma, malignancy, dehydration, chronic inflammatory disease, drugs, or other. Systemic workup revealed a normal serum analysis, including coagulation factors. Brain magnetic resonance imaging (MRI) and MR angiography neither showed a parenchymal lesion nor artery stenosis/occlusion. We performed a standard electroencephalogram (EEG) to differentiate epilepsy as a probable diagnosis, but no epileptic activity was detected. He received a diagnosis of kinetic tremor based on the characteristics of his attacks. Arotinolol was administrated initially and subsequently switched to clonazepam. However, the paroxysmal tremor continuously persisted with exacerbations.

One year later, the patient suffered a sudden weakness of the right extremities, verbal disturbance, and sensory deficit on the right side of his body. Initial MRI and MR angiography revealed no responsible lesion. An EEG showed no evidence of epilepsy. He was admitted to our service with a suspicion of cerebral ischemia and antiplatelet therapy was started. The tremor did not occur after hospitalization. On day 5, the right paralysis deteriorated. MRI showed dilatation of vessels, from deep medullary veins toward superficial medullary veins on the left frontal/parietal lobe and on the right frontal/parietal lobe (Fig. 2). White matter changes surrounding these dilated veins were observed as well. In contrast, there were no changes in bilateral basal ganglia, thalamus, brainstem and cerebellum. MR venography proved occluded superior sagittal sinus in the anterior part (Fig. 3). The diagnosis of cerebral venous thrombosis (CVT) was established and anticoagulation by intravenous low-molecular-weight heparin was immediately initiated. He refused to undergo any contrast studies, including cerebral angiography. Ten days later, he had a partial seizure starting in his right upper limb and passing over to the left upper limb and face. Brain computed tomography detected cortical hemorrhage in the left frontal lobe where gradual dilatation of cortical veins had been observed. Multiple cortical hemorrhages recurred with subsequent complication of obstructive hydrocephalus. Despite decompressive surgery and ventriculoperitoneal shunt placement, the patient stayed in coma and died of disseminated intravascular coagulation and multiple-organ failure induced by shunt infection on the 90th hospital day.

\section{Discussion}

We presumed that these attacks were due to kinetic tremor since apparently the attacks were triggered by actions. Although focal seizure was the most probable alternative explanation, epilepsy was ruled out by the absence of epileptic discharge on repeated EEGs. Moreover, the attack was different from convulsion since trembling was restricted to the distal portion of the extremities. Therefore, he was able to write with his affected hand even during the ictus.

In CVT, transient repetitive movement disorder is extremely rare except as a complication of epilepsy. Only 2 articles addressing involuntary movement complicated with CVT were found in a literature search. One was a case report in Japanese that presented a patient of CVT in the superior sagittal sinus initially manifesting postural tremor and headache 


\section{Case Reports in Neurology}

Murao et al.: Cerebral Venous Thrombosis in the Superior Sagittal Sinus as a Rare Cause of a Paroxysmal Kinetic Tremor

without parenchymal lesions [1]. The involuntary movement was unlikely to be epilepsy since epileptic abnormality was not detected on EEG. Cerebral angiography demonstrated occluded superior sagittal sinus in the anterior part with the development of collateral cortical veins. The authors did not examine the mechanism of postural tremor in this case. Therefore, the interaction between tremor and CVT was unclear. The other article reported the results of the VENOPORT study investigating clinical aspects and medical management of CVT using the retrospective and prospective cohort in Portugal. Only 1 out of 142 CVT patients experienced involuntary movement. However the details of this case were not mentioned [2].

In the present case, thalamus, basal ganglia, or cerebellum, which is known as a lesion responsible for various movement disorders [3,4], was not visibly affected. The current advances in functional brain imaging have contributed to a better pathophysiological understanding of movement disorders as complex alterations of widespread functional brain networks. Induced basal ganglia lesions caused by defects in functional connectivity of the basal ganglia-thalamocortical circuit contribute more to most movement disorders rather than to a localized brain lesion [5, 6]. Impairment of this functional brain network induced by intermittent dysfunction of left frontal cortex may have been the underlying mechanism. However, because of the lack of hemodynamic and functional evaluation using SPECT or PET, it is impossible to clarify the causal anatomical lesion.

The absence of focal neurological deficit delayed the diagnosis in the present case since transient neurological impairments are uncommon in CVT. The precise mechanism of transient neurologic disorder in CVT is undetermined. When the parenchymal lesion is absent, perfusion failure induced by venous congestion and elevated venous pressure may result in cerebral dysfunction [7]. Neurological symptoms may occur as a consequence of intermittent cerebral ischemia or venous congestion resolved by partial recanalization or by unstable collateral formation. Cortical and bridging veins are important to maintain venous drainage through collateral veins directing toward the superior sagittal sinus, hence, when these veins are affected, irreversible change may arise. In a previous study reviewing 38 cases of CVT, the authors reported that occlusion of the cerebral veins was related to focal neurological signs but not to isolated sinus occlusion [8]. In the present case, the cerebral cortical veins seem to have been patent in the early period since dilatation of the veins was not apparent. Probably because the cortical veins were spared, persistent focal neurological deficit may not have manifested at the beginning. We could not confirm this hypothesis since a precise serial evaluation of the development of collateral veins and the extent of the thrombus by cerebral angiography was not accomplished.

Since tremors were restricted to the right extremities, dysfunction of the left basal ganglia may have played a role. However, considering that cortical veins in the left frontal cortex had been gradually dilated and resulted in venous hemorrhage, functional impairment of the basal ganglia-thalamocortical circuit in the left frontal lobe induced by venous congestion and elevated venous pressure is a presumed pathophysiology.

\section{Conclusion}

To the best of our knowledge, this is the first report describing a patient with CVT with a 1-year history of paroxysmal kinetic tremor. We have to be aware that CVT can cause involuntary movement not associated with epilepsy. 
Murao et al.: Cerebral Venous Thrombosis in the Superior Sagittal Sinus as a Rare Cause of a Paroxysmal Kinetic Tremor

\section{Statement of Ethics}

Informed consent was obtained from a close relative of the patient.

\section{Disclosure Statement}

The authors have no conflicts of interest to disclose.

\section{References}

1 Nakaso K, Shimoda M, Yasui K, Mori M, Wakutani Y, Takeshima T, Nakashima K: A case of superior sagittal sinus thrombosis following long-term medication with carbamazepine (in Japanese). Rinsho Shinkeigaku 2000;40:617-620.

-2 Ferro JM, Correia M, Pontes C, Baptista MV, Pita F; Cerebral Venous Thrombosis Portuguese Collaborative Study Group (Venoport): Cerebral vein and dural sinus thrombosis in Portugal: 19801998. Cerebrovasc Dis 2001;11:177-182.

-3 Ghika-Schmid F, Ghika J, Regli F, Bogousslavsky J: Hyperkinetic movement disorders during and after acute stroke: the Lausanne Stroke Registry. J Neurol Sci 1997;146:109-116.

$\checkmark 4$ Kim JS: Delayed onset mixed involuntary movements after thalamic stroke: clinical, radiological and pathophysiological findings. Brain 2001;124:299-309.

-5 Holtbernd F, Eidelberg D: Functional brain networks in movement disorders: recent advances. Curr Opin Neurol 2012;25:392-401.

6 Mehanna R, Jankovic J: Movement disorders in cerebrovascular disease. Lancet Neurol 2013;12:597608.

7 Shinohara Y, Takagi S, Kobatake K, Gotoh F: Influence of cerebral venous obstruction on cerebral circulation in humans. Arch Neurol 1982;39:479-481.

-8 Bousser MG, Chiras J, Bories J, Castaigne P: Cerebral venous thrombosis - a review of 38 cases. Stroke 1985;16:199-213.
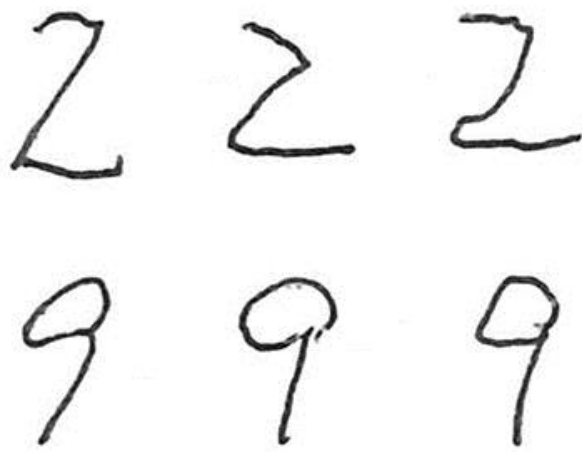

a
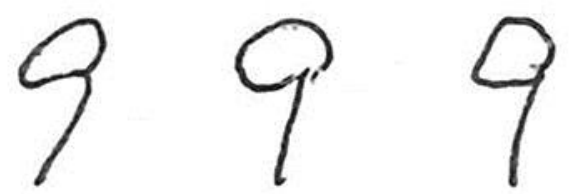
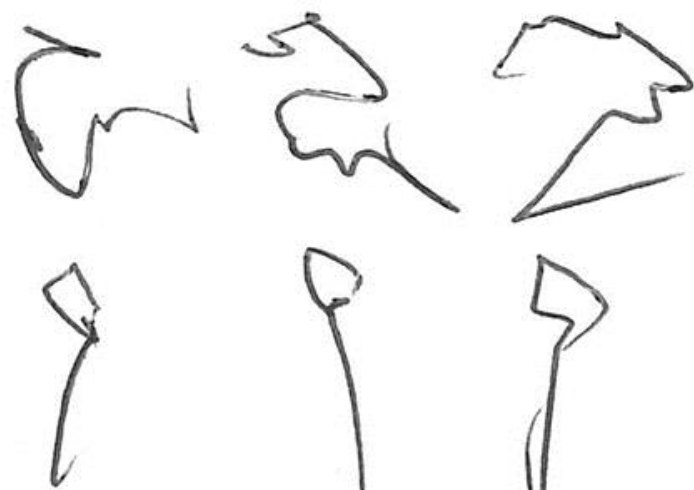

b
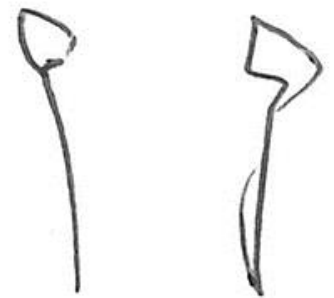

Fig. 1. Hand writing during the interictal period (a) and during the attack (b). 


\section{Case Reports in Neurology}

\begin{tabular}{l|l}
\hline Case Rep Neurol 2016:8:276-281 \\
\hline DOI: 10.1159/000455022 & $\begin{array}{l}\text { C 2016 The Author(s). Published by S. Karger AG, Basel } \\
\text { www.karger.com/crn }\end{array}$ \\
\hline
\end{tabular}

Murao et al:: Cerebral Venous Thrombosis in the Superior Sagittal Sinus as a Rare Cause of a Paroxysmal Kinetic Tremor

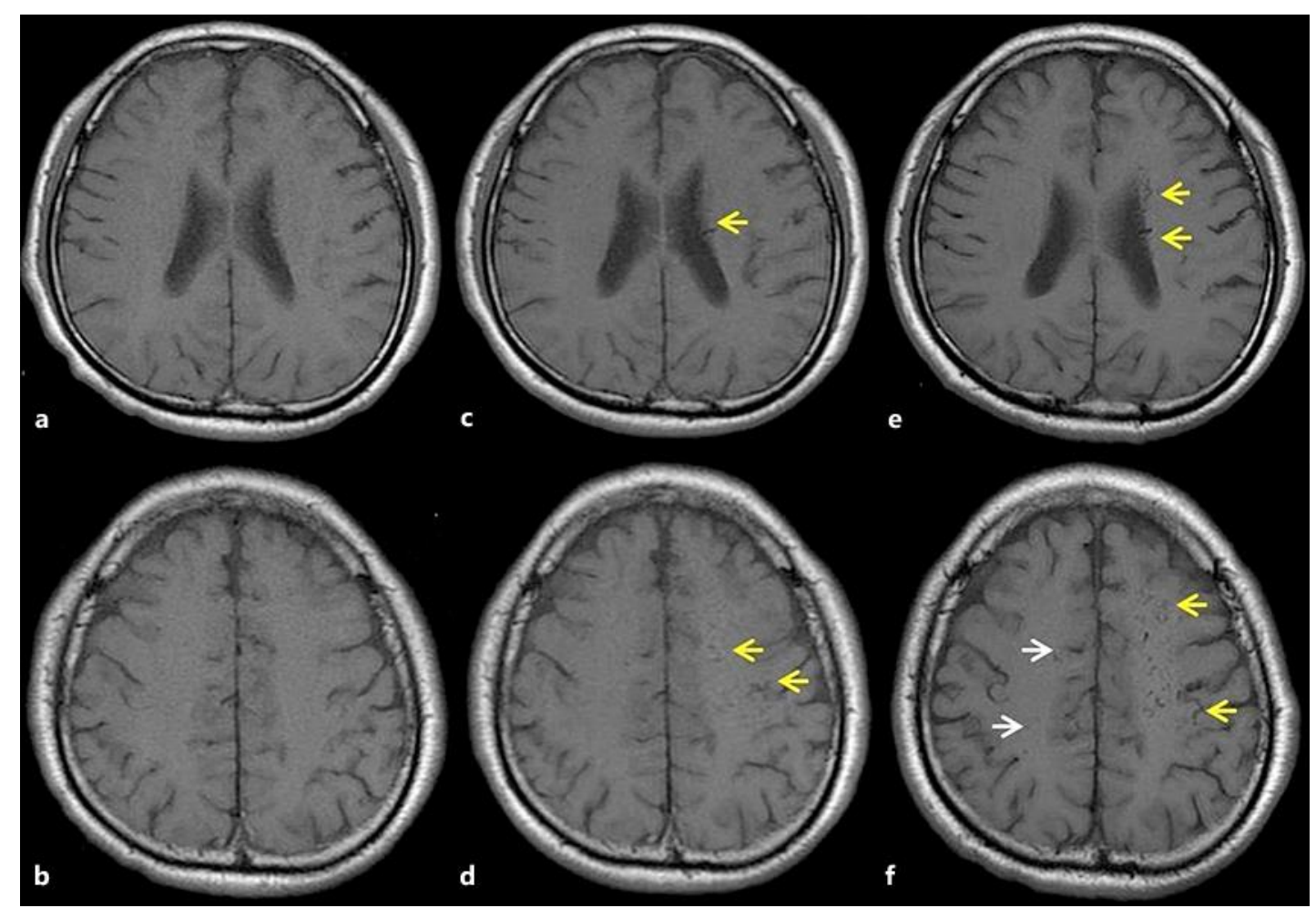

Fig. 2. Axial T1-weighted images (T1WI) showing gradual dilatation of the superficial medullary veins on the left frontal/parietal lobe (yellow arrows) and on the right frontal and parietal lobe (white arrows) during the clinical course. $\mathbf{a}, \mathbf{b}$ T1WI performed at the first visit when complaining of a jerk attack. c, d T1WI obtained at admission to the hospitalization. e, f T1WI taken at neurological deterioration during hospitalization. 


\section{Case Reports in Neurology}

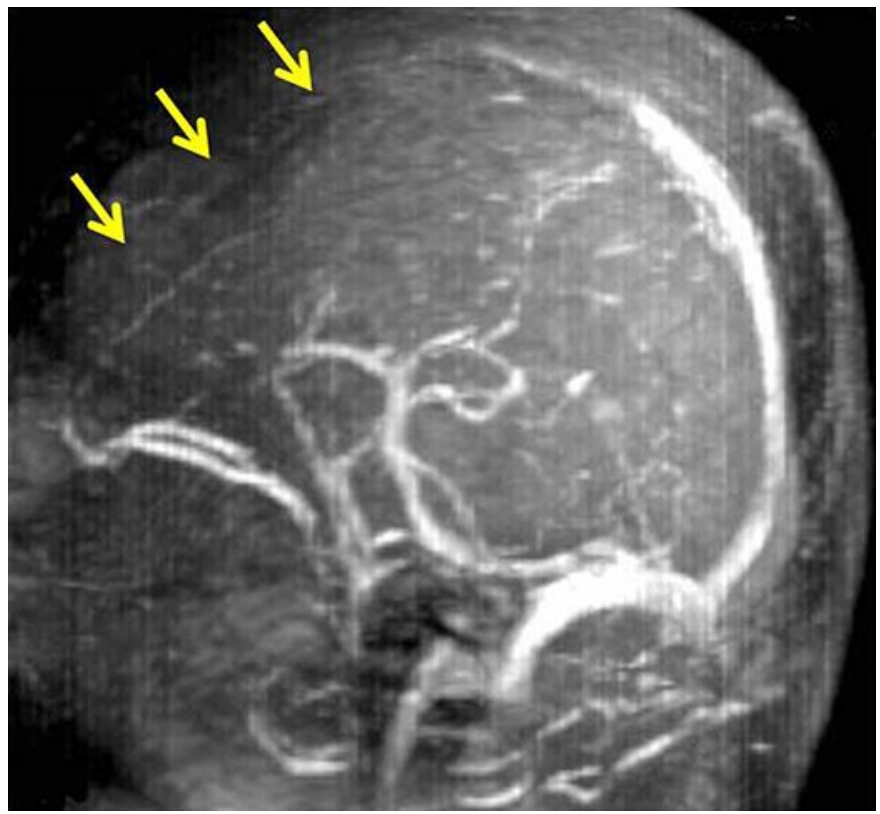

Fig. 3. MR venography at neurological deterioration during hospitalization presents an occluded superior sagittal sinus (yellow arrows).

Murao et al.: Cerebral Venous Thrombosis in the Superior Sagittal Sinus as a Rare Cause of a Paroxysmal Kinetic Tremor 